\title{
Relação da maturação com a antropometria e aptidão física na iniciação desportiva
}

\author{
Relationship of maturation with anthropometric and physical valences in \\ sports initiation boys and girls
}

\author{
B.G. Cabral, S.A. Cabral, R.M. Medeiros, T. Alcatara, P.M.S. Dantas
}

ARTIGO ORIGINAL | ORIGINAL ARTICLE

\begin{abstract}
O presente estudo centra-se em verificar a influência da maturação na antropometria e aptidão física de jovens praticantes de voleibol, observando a correlação do estado maturacional com as demais variáveis. A amostra do estudo foi composta por 149 sujeitos $(n=149)$ de 8 a 14 anos. Para verificação da maturação, utilizou-se a idade cronológica e idade óssea como preditor maturacional através do método Grave-Brown (1976). Por sua vez, para as variáveis antropométricas, foram usadas as medidas de massa, estatura, índice ponderal, diâmetros biacromial e bi-crista ilíaca, perímetros de braço e perna, e perímetros de braço e de perna corrigidos, além dos testes físicos de agilidade, coordenação e força explosiva de membros inferiores e superiores. Ao observar a correlação entre a maturação e as demais variáveis, constatou-se que, no feminino, apenas o índice ponderal não apresentou correlação significativa e, no masculino, o índice ponderal, agilidade e coordenação. Em ambos os sexos, a estatura e diâmetros biacromial e bi-crista ilíaca apresentaram bons resultados. Assim, o estudo permite concluir que existe correlação entre o estágio maturacional, a variável força e variáveis antropométricas, ressaltando, ainda, a importância da avaliação de diferentes variáveis ao se trabalhar com orientação e promoção de talentos no desporto.

Palavras-chave: idade óssea, morfologia, desporto
\end{abstract}

ABSTRACT

The aim of this study was to verify the influence of maturation in anthropometry and physical valences of young volleyball players, assessing the correlation between maturational state and other variables. The sample was composed of 149 children between 8 and 14 years. Chronological age and bone age as maturational predictor with the method of Grave-Brown (1976) were selected to assess the maturation state. As anthropometrics weight, height, ponderal index, biacromial diameter and bi-iliocristal diameter, perimeter of arm and leg, and perimeter of leg fixed were measured. In addition, it was also assessed the physical agility tests, coordination and explosive force of lower and upper limbs. All but ponderal index presents significant correlation with maturational state in girls. For boys, all correlations were significant except the ponderal index, agility and coordination. In both genders, the height and biacromial and bi-iliocristal diameter presented strong associations, allowing us to complete a correlation in maturational stage with the variable strength and anthropometric variables, as well as allows us to confirm the importance of evaluation from different variables when working with guidance and promotion of talent in the sport.

Keywords: bone age, morphology, sports

Submetido: 21.05.2012 | Aceite: 30.05.2013

Breno Guilherme Cabral, Suzet de Araujo Cabral, Radamés Marciel Medeiros, Paulo Moreira Silva Dantas. Departamento de Educação Física, Universidade Federal do Rio Grande do Norte, Brasil.

Tabata Alcatara. Universidade Potiguar - UNP, Rio Grande do Norte, Brasil.

Endereço para correspondência: Breno Guilherme Cabral, Departamento de Educação Física, Universidade Federal do Rio Grande do Norte, Caixa Postal 1524 - Campus Universitário Lagoa Nova, CEP 59078-970 Natal/RN - Brasil.

E-mail: brenotcabral@gmail.com 
No trabalho desportivo com jovens atletas o estado maturacional tem sido um importante fator a ser observado, sendo um conjunto de processos dinâmicos associados a um amplo aspeto de alterações morfológicas, orgânicas e de desenvolvimento, que apresenta variações individuais de velocidade durante o processo (Linhares et al., 2009; Malina et al., 2006).

Nesse contexto, os estudos da ciência desportiva têm evidenciado a grande importância da observação de diferentes variáveis e suas relações de interferência na determinação de características individuais que, em grande parte, estão diretamente ligadas ao desenvolvimento desportivo. Entre essas variáveis podemos citar: as antropométricas, as qualidades físicas, a velocidade de maturação e a variabilidade genética, onde as relações de interferências entre as variáveis são consideradas fatores determinantes no processo de desenvolvimento (Malina \& Bouchard, 2002; Matsudo, Araujo, Oliveira, \& Rodrigues, 2007).

A orientação desportiva, quando feita adequadamente, permite relacionar características individuais a necessidades específicas de cada modalidade desportiva, proporcionando ao indivíduo a possibilidade de progredir, quando as suas características físicas são potencializadas a partir do conhecimento desses elementos de determinação do seu potencial de desempenho, observando, também, a influência dos fatores de interferência como o estado maturacional (Linhares et al., 2009; Mohamed et al., 2009).

A partir desse pressuposto, surge a necessidade de utilização de métodos no processo de evolução científica do desporto que permitam observar o indivíduo, a partir de um diagnóstico antecipado de suas habilidades e características individuais. Diante do exposto o presente estudo centra-se no objetivo de analisar a correlação de variáveis físicas e antropométricas com a maturação em jovens atletas.

\section{Participantes}

\section{MÉTODo}

O estudo é do tipo descritivo com tipologia transversal, onde foi investigada uma amostra total de cento e quarenta e nove sujeitos ( $\mathrm{n}=$ 149), sendo 55 do sexo masculino e 94 do sexo feminino, com idades entre 8 e 14 anos, na cidade do Natal/RN, escolhidos de forma não probabilística intencional entre alunos de um projeto social, que atua apenas com iniciação desportiva no Voleibol, com uma frequência de três sessões semanais de uma hora (1h) de treino. Foram definidos como critérios de exclusão: apresentar problemas de saúde que possam interferir no desenvolvimento dos testes, não concordar com a realização dos testes ou por motivos diversos interromper os testes por mais de 10 dias.

Todos os protocolos usados na presente investigação seguiram rigorosamente as normas de ética em pesquisa, respeitando os itens propostos pela resolução 196/96 CNS-Brasil e Declaração de Helsínquia de 1975. Os procedimentos realizados na presente pesquisa foram autorizados pelo comitê de ética local de acordo com o parecer $\mathrm{n}^{\circ}$ 071/071/2010.

\section{Instrumentos}

Para observação da variável aptidão física foi usado para verificar a força explosiva de membros inferiores uma régua graduada em centímetros, fixada em uma parede a partir de 2.00 metros, propiciando a realização dos movimentos preparatórios em um "vão livre". Para a força explosiva de membros superiores foram usadas uma cadeira, uma fita métrica da marca Sanny e uma bola de medicineball de $2 \mathrm{~kg}$. Para a velocidade de membro superiores foram usados 2 discos de borracha de $20 \mathrm{~cm}$ de diâmetro fixados horizontalmente a uma mesa, distantes $80 \mathrm{~cm}$ um do outro e entre eles um retângulo de borracha $(10 \times 20 \mathrm{~cm})$. Para a agilidade foram usadas as linhas de um campo oficial de Voleibol e um cronómetro Samsung 520.

$\mathrm{Na}$ verificação das variáveis antropométricas foram utilizados os seguintes instrumentos: uma balança eletrônica Filizola-110 para a massa corporal, com capacidade para $150 \mathrm{~kg}$ e divisões de 1/10 de kg, um estadiómetro Sanny ES2020 para estatura, uma fita antropométrica Sanny (precisão de $0.1 \mathrm{~mm}$ ) para aferição dos 
perímetros, um paquímetro pequeno Sanny para os diâmetros e um compasso de dobras cutâneas científico Sanny, de fabricação brasileira, para aferir as dobras.

A determinação da idade óssea foi realizada por meio de um aparelho da marca Rhos, com ampola Toshiba de $60 \mathrm{Kvp}$ e $9 \mathrm{~mA}$, a uma distância foco-filme de $75 \mathrm{~cm}$. O filme utilizado foi Kodak TMATX de $18 \times 24 \mathrm{~cm}$, em chassi com ecrã de terras raras, revelados em uma processadora automática Dentxr, com componentes químicos para Kodak.

\section{Procedimentos}

Sendo o processo maturacional um processo contínuo, a realização dos testes para análise das variáveis antropométricas, idade óssea (através de raio $\mathrm{X}$ ) e de aptidão física, ocorreram com intervalo máximo de 10 dias, evitando dessa forma interferência do tempo.

$\mathrm{Na}$ tentativa de minimizar ao máximo quaisquer interveniências na mensuração das medidas antropométricas, houve o cuidado de observar o erro técnico de medida (ETM) intraavaliador, uma vez que todas as medidas foram realizadas pelo mesmo avaliador. Observou-se o intervalo de $0.7 \%$ a $4.6 \%$ de ETM e $9.52 \%$ a $43.25 \%$ no coeficiente de variação (Perini, Oliveira, Ornellas, \& Oliveira, 2005).

\section{Aptidão Física}

Força explosiva de membros inferiores (FEMI): Para verificação da força explosiva dos membros inferiores, foi utilizado o Sargent Jump Test, no qual o testado ficou em pé, mantendo as plantas dos pés em contato com o solo, de lado para a superfície graduada, com o braço estendido acima da cabeça, onde foi anotada a altura máxima. Após uma semiflexão dos joelhos, o testado realiza uma rápida transição excêntrica/concêntrica e salta o mais verticalmente possível tocando a régua com a ponta dos dedos, os quais foram previamente marcados com pó de giz. O testado saltou três vezes consecutivas, sendo considerado o maior salto. $\mathrm{O}$ resultado foi dado em $\mathrm{cm}$ diminuindo-se a altura alcançada com salto pela altura máxima alcançada (Marins \& Giannichi, 2003).

Força explosiva de membros superiores (FEMS): Foi utilizado o teste de arremesso de medicineball, para avaliar a força explosiva de membros superiores. O testado ficou sentado em uma cadeira, preso por uma corda na altura do peito para evitar o embalo durante o arremesso, segurando a bola de medicineball de 2 $\mathrm{kg}$ com as duas mãos contra o peito, no qual, arremessou a bola o mais longe possível. Foram realizadas três tentativas consecutivas e registada a melhor marca. A fita métrica foi colocada no chão a partir das pernas dianteiras da cadeira, sendo medida a distância entre o ponto inicial e o primeiro ponto de contato da bola com o solo (Gaya \& Silva, 2007).

Velocidade de membros superiores: Para observação da velocidade de membros superiores, foi utilizado o teste de golpeio de placas, onde o testado deverá ficar em pé, em frente a uma mesa com altura regulável na altura da cintura. Sobre a mesa foram colocados 2 discos a uma distância de 80 centímetros com um retângulo entre os mesmos, todos fixados horizontalmente a mesa. A mão não dominante do testado ficou parada em cima do retângulo e a mão dominante no círculo oposto a lado da mesma. Ao sinal, sem mexer a mão do retângulo, o testado realizou 25 ciclos batendo com a mão dominante no outro círculo e voltando ao inicial com a maior velocidade possível. Cada vez que uma das placas não fosse batida o avaliador deveria aumentar um ciclo. Cada testando devera realizar duas tentativas, sendo registrado o menor tempo entre as tentativas realizadas. Esse protocolo faz parte da bateria de testes EUROFIT e deve ser realizado com um avaliador para contagem do tempo e outro para contagem dos ciclos (Conselho da Europa, 1990).

Agilidade: Para avaliar a agilidade foi utilizado o teste de 30 metros, onde o testado correu em velocidade máxima a partir da linha de serviço até a linha dos três metros do campo de voleibol, retornou à linha inicial (linha de serviço), correu até a linha de serviço do campo oposto a que iniciou o teste o mais rápido pos- 
sível, passando pela linha sem diminuir a velocidade, para que o tempo fosse computado no momento em que cruzou a última linha (Buligin, 1981). Foram realizadas duas tentativas com intervalo de 5 minutos e registado o melhor tempo.

\section{Antropometria}

Os sujeitos foram mensurados descalços e usando roupas leves.

Massa corporal: Com o indivíduo em pé, no centro da plataforma da balança, a massa foi registada em quilogramas.

Estatura corporal: É a distância entre a planta dos pés e o ponto mais alto da cabeça (vértex), em apneia, com a cabeça orientada no plano de Frankfurt, registrada em centímetros.

Índice ponderal (IP): O índice ponderal foi medido a partir do resultado da divisão da estatura pela raiz cúbica da massa corporal.

Perímetros e diâmetros: Para aferir os perímetros e diâmetros foram seguidos os procedimentos de Marfell-Jones, Olds, Stewart e Carter (2006). Foram aferidos o Perímetro do Braço $(\mathrm{Pb})$, Perímetro corrigido do Braço (Pcb) que se refere ao perímetro do braço em centímetros, subtraído pelo valor da dobra cutânea tricipital (TR) transformado em $\mathrm{cm}$, Perímetro de perna ( $\mathrm{Pp}$ ) e o Perímetro corrigido de perna (Pcp) que se refere ao perímetro de perna (panturrilha) em centímetros, subtraído pelo valor da dobra cutânea de perna transformado em cm, Diâmetro bi-cristailíaco (Di.bi.cr) e Diâmetro biacromial (Di.bi.ac).

Dobras cutâneas: As medidas de dobras cutâneas foram realizadas no lado direito dos sujeitos e repetidas três vezes sucessivas em cada local seguindo o protocolo Marins e Giannichi (2003), sendo utilizada a média como valor da medida ou dois valores coincidentes. Foram aferidas a Dobra tricipital (TR) e a Dobra de perna (PM).

\section{Idade Cronológica}

Para determinação precisa da idade cronológica de um indivíduo, foi contada a quantidade de meses de vida do mesmo, a partir de sua data de nascimento com dia, mês e ano e dividida por 12 .

\section{Idade Óssea}

Na determinação da idade óssea foi utilizado o método Grave e Brown (1976), onde a partir do raio $\mathrm{X}$ de mão e punho do indivíduo, a idade óssea foi determinada por laudo médico, observando a sequência de eventos da ossificação do indivíduo através de comparação com o atlas proposto por Pyle, Waterhouse e Greulich (1971). Na realização do exame foi administrada de forma individualizada uma única dosagem radiográfica, a uma distância foco-filme de $75 \mathrm{~cm}$. As películas radiográficas foram analisadas de forma independente por 3 radiologistas, sendo os resultados dos laudos dos mesmos testados estatisticamente através do método Kappa, tendo se obtido uma concordância inter-avaliador de .88 , a qual que confirma a fiabilidade das análises.

\section{Maturação}

O componente maturação classifica os indivíduos em estado maturacional atrasado, normal ou acelerado, sendo essa classificação determinada a partir da subtração da idade óssea do indivíduo em meses, pela idade cronológica do mesmo em meses. Realizado o cálculo, quando o indivíduo apresenta entre + 12 e -12 em relação à soma de meses da idade cronológica é considerado normal, acima de +12 é considerado acelerado e abaixo de -12 é considerado atrasado em relação a sua idade cronológica (Malina \& Bouchard, 2002).

\section{Análise Estatística}

Para o tratamento estatístico dos dados foi utilizado o programa Stata for Windows, versão 10.0. A análise estatística utilizada foi à descritiva, estabelecendo medidas de tendência central e dispersão na busca de construir um perfil de características dos grupos, formados pelos indivíduos do sexo feminino e masculino, nas variáveis investigadas, uma vez que foi observada diferença significativa entre os grupos. O pressuposto de distribuição normal 
dos dados foi verificado através do teste de Kolmogorov-Smirnov e, posteriormente, foram calculados os coeficientes de correlação de Pearson entre a maturação e as variáveis antropométricas e de aptidão física.

O nível de significância para estabelecido em $p<.05$ ou $95 \%$ de probabilidade.

\section{RESULTADOS}

Verifica-se na tabela 1 que, comparativamente ao sexo masculino, existe uma considerável prevalência de indivíduos do sexo feminino no grupo estudado (63.08\%), sendo o grupo feminino composto, em sua maioria, por meninas com maturação acelerada em relação à sua idade cronológica. Em sentido diverso, no grupo do sexo masculino, evidencia-se que a minoria é integrada por indivíduos de maturação acelerada, tendo sua maioria formada por aqueles de maturação normal em relação à sua idade cronológica.

Impõe-se destacar que a variável maturação revelou valores distintos entre o sexo feminino e masculino, apresentando uma diferença significativa $(p<.001)$, a qual justifica o estudo das variáveis separadas por sexo.
Tabela 1

Caracterização do grupo em média, desvio padrão e percentuais

\begin{tabular}{|c|c|c|}
\hline & $\begin{array}{l}\text { Masculino } \\
(\mathrm{n}=55)\end{array}$ & $\begin{array}{l}\text { Feminino } \\
(\mathrm{n}=94)\end{array}$ \\
\hline Maturação $(\mathrm{M} \pm \mathrm{DP})$ & $-0.05 \pm 1.48$ & $1.02 \pm 1.55$ \\
\hline Idade óssea $(\mathrm{M} \pm \mathrm{DP})$ & $12.11 \pm 2.08$ & $13.43 \pm 2.53$ \\
\hline $\begin{array}{l}\text { Idade cronológica } \\
(\mathrm{M} \pm \mathrm{DP})\end{array}$ & $12.17 \pm 1.42$ & $12.41 \pm 1.52$ \\
\hline Atrasados (\%) & 20 & 7.45 \\
\hline Normais (\%) & 65.45 & 38.30 \\
\hline Acelerados (\%) & 14.55 & 54.25 \\
\hline Gênero (\%) & 36.92 & 63.08 \\
\hline
\end{tabular}

Em relação à tabela 2, analisando-se, especificamente, a correlação da maturação com aptidão física e antropometria, observaram-se correlações significativas em muitas das variáveis observadas.

Nos dados coletados no masculino, não foi encontrada correlação significativa apenas para a agilidade, coordenação e índice ponderal. Por outro lado, a força explosiva de membros inferiores, diâmetro bicristailíaca, perímetros de braço e de perna, e perímetro corrigido de braço apresentaram moderados índices de

Tabela 2

Correlação da maturação com a antropometria e aptidão física

\begin{tabular}{lrrrr}
\hline & \multicolumn{2}{c}{$\begin{array}{c}\text { Masculino } \\
(\mathrm{n}=55)\end{array}$} & \multicolumn{2}{c}{$\begin{array}{c}\text { Feminino } \\
(\mathrm{n}=94)\end{array}$} \\
\cline { 2 - 5 } & \multicolumn{1}{c}{$p$} & \multicolumn{1}{c}{$r$} & \multicolumn{1}{c}{$p$} \\
\hline Agilidade & -.141 & .300 & -.285 & .010 \\
Força explosiva membros superiores & .530 & $<.001$ & .585 & $<.001$ \\
Força explosiva membros inferiores & .342 & .010 & .227 & .030 \\
Coordenação & -.262 & .053 & -.381 & $<.001$ \\
Massa corporal & .526 & $<.001$ & .654 & $<.001$ \\
Estatura & .812 & $<.001$ & .716 & $<.001$ \\
Índice Ponderal & .146 & .289 & -.180 & .077 \\
Distância biacromial & .657 & $<.001$ & .666 & $<.001$ \\
Distância bicristailíaca & .657 & $<.001$ & .523 & $<.001$ \\
Perímetro braço & .427 & .001 & .506 & $<.001$ \\
Perímetro perna & .405 & .002 & .570 & $<.001$ \\
Perímetro corrigido de braço & .479 & $<.001$ & .533 & $<.001$ \\
Perímetro corrigido de perna & .545 & $<.001$ & .602 & $<.001$ \\
\hline
\end{tabular}


Tabela 3

Valores descritivos $(M \pm D P)$ das variáveis antropométricas, aptidão física e idade cronológica de acordo com o estágio maturacional por sexo

\begin{tabular}{|c|c|c|c|c|c|c|}
\hline & \multicolumn{2}{|c|}{ Maturação atrasada } & \multicolumn{2}{|c|}{ Maturação normal } & \multicolumn{2}{|c|}{ Maturação acelerada } \\
\hline & $\begin{array}{l}\text { Masculino } \\
(\mathrm{n}=11)\end{array}$ & $\begin{array}{c}\text { Feminino } \\
(\mathrm{n}=7)\end{array}$ & $\begin{array}{c}\text { Masculino } \\
(\mathrm{n}=36)\end{array}$ & $\begin{array}{c}\text { Feminino } \\
(\mathrm{n}=36)\end{array}$ & $\begin{array}{l}\text { Masculino } \\
\quad(\mathrm{n}=8)\end{array}$ & $\begin{array}{c}\text { Feminino } \\
(\mathrm{n}=51)\end{array}$ \\
\hline IC & $12.4 \pm 1.4$ & $11.9 \pm 1.4$ & $12.0 \pm 1.5$ & $11.8 \pm 1.7$ & $12.5 \pm 1.1$ & $12.9 \pm 1.2$ \\
\hline Agil & $12.0 \pm 1.2$ & $13.3 \pm 1.2$ & $12.5 \pm 1.3$ & $13.3 \pm 1.4$ & $12.8 \pm 1.9$ & $13.1 \pm 1.0$ \\
\hline FEMS & $1.8 \pm 0.6$ & $1.7 \pm 0.5$ & $2.4 \pm 0.8$ & $2.0 \pm 0.8$ & $2.6 \pm 1.5$ & $2.5 \pm 0.6$ \\
\hline FEMI & $0.3 \pm 0.1$ & $0.3 \pm 0.1$ & $0.3 \pm 0.1$ & $0.3 \pm 0.1$ & $0.3 \pm 0.1$ & $0.3 \pm 0.1$ \\
\hline Coor & $16.4 \pm 3.0$ & $18.9 \pm 2.5$ & $17.3 \pm 3.2$ & $17.1 \pm 2.2$ & $17.1 \pm 3.6$ & $16.4 \pm 2.3$ \\
\hline Massa & $37.3 \pm 6.5$ & $36.3 \pm 3.8$ & $45.7 \pm 15.2$ & $41.9 \pm 11.9$ & $58.2 \pm 9.4$ & $51.6 \pm 11.7$ \\
\hline Estat. & $1.5 \pm 0.1$ & $1.4 \pm 0.1$ & $1.5 \pm 0.1$ & $1.5 \pm 0.1$ & $1.6 \pm 0.1$ & $1.6 \pm 0.1$ \\
\hline IP & $42.5 \pm 3.5$ & $42.5 \pm 2.6$ & $42.4 \pm 2.8$ & $43.2 \pm 2.6$ & $44.0 \pm 2.4$ & $42.5 \pm 2.6$ \\
\hline D.bi.ac & $32.2 \pm 2.3$ & $32.0 \pm 1.2$ & $34.0 \pm 3.4$ & $33.2 \pm 3.3$ & $36.5 \pm 2.4$ & $35.3 \pm 2.8$ \\
\hline D.bi.cr & $25.6 \pm 1.6$ & $22.7 \pm 1.2$ & $24.3 \pm 3.4$ & $23.8 \pm 3.7$ & $21.8 \pm 2.6$ & $25.0 \pm 2.3$ \\
\hline $\mathrm{Pb}$ & $21.8 \pm 2.3$ & $22.6 \pm 2.3$ & $24.6 \pm 3.9$ & $22.7 \pm 3.4$ & $21.8 \pm 2.3$ & $25.1 \pm 3.6$ \\
\hline $\mathrm{Pp}$ & $29.0 \pm 2.1$ & $29.2 \pm 1.5$ & $31.3 \pm 4.5$ & $30.4 \pm 3.7$ & $34.6 \pm 3.1$ & $32.9 \pm 3.7$ \\
\hline Pcb & $26.5 \pm 3.8$ & $21.1 \pm 2.0$ & $23.2 \pm 3.3$ & $21.3 \pm 3.0$ & $20.6 \pm 1.9$ & $23.5 \pm 3.1$ \\
\hline Pcp & $27.5 \pm 1.8$ & $27.7 \pm 1.8$ & $30.0 \pm 4.2$ & $28.8 \pm 2.8$ & $33.1 \pm 2.6$ & $31.1 \pm 3.2$ \\
\hline
\end{tabular}

Nota: IC-idade cronológica (anos); Agil- agilidade (seg); FEMS- Força explosiva de membros superiores (m); FEMI- força explosiva de membros inferiores(m); Coor.- Coordenação motora (seg); Estat.- Estatura (m); IP- Índice ponderal; D.bi.acdistancia biacromial $(\mathrm{cm})$; D.bi.cr- distancia bicristailíaca $(\mathrm{cm})$; Pb- Perímetro de braço $(\mathrm{cm})$; Pp- Perímetro de perna $(\mathrm{cm})$; Pcb- Perímetro corrigido de braço $(\mathrm{cm})$; Pcp- Perímetro corrigido de perna $(\mathrm{cm})$; Massa $(\mathrm{kg})$.

correlação com o momento maturacional do sexo masculino.

A força explosiva de membros superiores, massa corporal, diâmetros biacromial e bicristailíaca, e perímetro corrigido de perna expressaram níveis de significância para correlação ainda melhores e diâmetro biacromial, sendo a estatura a variável que manifestou melhor resultado em relação à maturação com uma alta correlação.

Para o sexo feminino, apenas o índice ponderal não apresentou correlação, sendo a agilidade, força explosiva de membros inferiores e coordenação as variáveis com menores níveis de correlação. Neste ponto, cumpre observar que todas as demais variáveis analisadas apresentam bons índices de correlação com a maturação dos indivíduos estudados, sendo, mais uma vez, a estatura a variável que manifestou resultado mais elevado.
A tabela 3 permite uma melhor análise dos dados inseridos de forma descritiva, onde os indivíduos foram separados por sexo de acordo com o momento maturacional, sendo aqueles tidos como acelerados os que apresentaram idade óssea maior que a idade cronológica. Por sua vez, os atrasados manifestaram idade óssea menor que a idade cronológica e os normais não demonstraram diferença significativa entre a idade óssea e a idade cronológica.

\section{DISCUSSÃO}

Os resultados encontrados (ver tabela 2) demonstram que, tanto no masculino quanto no feminino, a estatura é a variável que apresenta maior correlação com a maturação. Segundo Malina, Bouchard e Bar-Or (2004), à medida que há um avanço no processo maturacional da criança, a tendência é que, com as mudanças fisiológicas e metabólicas, o indiví- 
duo atinja com maior velocidade a sua estatura final e, consequentemente, também se torne mais pesado devido ao amadurecimento fisiológico geral e aumento de massa corporal.

A estatura é um fator a ser analisado com muita atenção, observando as suas diferentes variáveis de influência como o estado maturacional, principalmente ao se tratar de orientação e desenvolvimento de jovens talentos desportivos em modalidades como o Voleibol que, segundo Gabbett, Georgieff, e Domrow (2007) e Cabral et al. (2008), apresenta a variável estatura como fator predominante para se alcançar o alto rendimento da modalidade.

$\mathrm{O}$ índice ponderal, que diz respeito à relação estatura e peso ou à linearidade do indivíduo, apresentou baixa correlação com a maturação nos sexos masculino e feminino. O referido resultado é considerado um dado relevante no processo de seleção e orientação de jovens no desporto, confirmando, mais uma vez, ser imprescindível a observação do indivíduo em suas diferentes variáveis. Assim, o indivíduo com maior linearidade não tem necessariamente maior predisposição a adquirir uma maior estatura. Segundo Schneider e Meyer (2005), a variável estatura dependerá da influência de uma série de outras variáveis, como, por exemplo, a maturação.

Ainda na tabela 2, verificou-se a correlação da maturação com os dados antropométricos, sendo além da estatura, os diâmetros biacromial e bicristailíaca, e o perímetro corrigido de perna, as variáveis que apresentam melhores resultados correlacionais em ambos os sexos, dados esses que corroboram a literatura, onde sabemos que diâmetros e espessuras corporais tendem a acompanhar o processo maturacional de forma positiva (Forwood et al., 2006; Malina, 2003).

A literatura afirma que a evolução do desempenho motor, na infância e na adolescência, está indiscutivelmente associada aos processos de crescimento e maturação. Diante de tal relação de interdependência, na avaliação do desempenho motor, urge que, ao se trabalhar com o desenvolvimento da criança no âmbito desportivo, sejam considerados os aspetos do crescimento físico e as idades cronológica e biológica (Gallahue \& Ozmun, 2005).

Ao avaliar a aptidão física no que se refere à maturação, as pesquisas têm observado um aumento nos valores de força proporcional aos estágios maturacionais, ou seja, quanto mais maturado o indivíduo, mais força ele tende a apresentar, devido a um aumento na massa muscular e à melhoria no desempenho motor $\mathrm{e}$ cognitivo (Biassio, Matsudo, \& Matsudo, 2004; Makarenko, 2001). Tal fato confirma os resultados obtidos na tabela 2, evidenciando uma correlação moderada da maturação com a força explosiva de membros inferiores e principalmente com a força explosiva de membros superiores em ambos os sexos, corroborando os estudos de Biassio et al. (2004) e Nedeljkovic, Mirkov, Kukolj, Ugarkovic e Jaric (2007).

Malina et al. (2004) e Pearson, Naughton e Torode (2006) defendem, ainda, que os aspetos referentes à maturação e à força têm grande importância no grande conjunto de fatores influenciadores da formação de atletas, atestando, portanto, a indispensabilidade de observação geral do indivíduo e não apenas fatores como a idade cronológica, uma vez que, durante a puberdade, a diferença entre os aspetos físicos de indivíduos de mesma idade cronológica podem ser significativas.

Ao analisar a tabela 3, verificamos que, para o sexo masculino, existiu uma concentração de indivíduos com maturação normal, o que pode ser explicado pelas fases diferenciadas de maturação destes em relação às mulheres. Foi visível, ainda, que, entre os estados maturacionais classificados, as médias para a idade cronológica encontram-se muito próximas, assim como as médias e desvio padrão da variável estatura, impondo-se a análise de mencionado dado, sobretudo pelo fato de que as médias das demais variáveis antropométricas tendem a ser crescentes para os indivíduos, acompanhando o seu estágio maturacional.

Especificamente, em se tratando de variáveis antropométricas, em ambos os sexos, as menores médias são visíveis em indivíduos 
com maturação atrasada. Por outro lado, são maiores para aqueles que se encontram no estágio normal e, ainda, maiores para os que se encontram com maturação acelerada sucessivamente. Tal fato permite visualizar o aumento da massa, crescimento e alargamento da estrutura corporal, acompanhando a evolução do processo maturacional do indivíduo, sem considerar apenas a idade cronológica ou estatura, ou ainda, a estatura e distâncias bicristailíaca e biacromial, guardando, portanto, consonância com os estudos de Latt et al. (2009) e Linhares et al. (2009).

Observou-se, ainda, na tabela 3 que o sexo feminino apresentou um comportamento diferente do masculino onde existe, nesse grupo, uma concentração maior de indivíduos com idade óssea superior à idade cronológica, caracterizando-se, assim, com maturação acelerada, fato esse também observado na tabela 1 , onde, ao analisar as médias de maturação nos meninos e meninas verificámos diferença significativa $(p<.001)$. Esses resultados corroboram as pesquisas científicas anteriores, comprovando que diferentes fatores podem influenciar na velocidade do processo de maturação, ressaltando, todavia, que, de maneira geral, o desenvolvimento maturacional das meninas tende a ser um processo mais acelerado em relação aos meninos (Kishali, Imamoglu, Katkat, Atan, \& Akyol, 2006; Little \& Malina, 2007).

Devido ao acelerado processo de maturação das meninas nessa faixa etária, também se pode explicar a diferença de estatura entre os três níveis maturacionais de forma crescente e positiva, onde em seus estudos, Roman, Ribeiro, Guerra Júnior e Barros Filho (2009) constataram que a idade da menarca influencia diretamente nos fatores antropométricos das meninas que, após a menarca, tendem a atingir até $95 \%$ de sua estatura da vida adulta.

\section{CONCLUSÕES}

Perante os resultados evidenciados, faz-se concluir que persiste correlação significativa entre o estágio de desenvolvimento maturacional da criança e adolescente, variáveis antro- pométricas e qualidades físicas como a força, sendo a força explosiva de membros superiores a variável de aptidão física que apresenta maior correlação positiva em ambos os sexos, consonante, porquanto, com a literatura atual.

A partir dos resultados obtidos, podemos confirmar a necessidade de, ao se analisar a promoção e desenvolvimento de talentos desportivos, considerar-se as diferentes variáveis, evitando, por via de consequência, o advento de erros, provocados, sobretudo, nas situações em que uma variável é analisada de forma independente, ao se ignorar fatores de forte influência e interferência entre as diferentes variáveis, como, por exemplo, o estágio maturacional.

\section{Agradecimentos:}

Nada a declarar.

\section{Conflito de Interesses:}

Nada a declarar.

\section{Financiamento:}

Nada a declarar.

\section{REFERÊNCIAS}

Biassio, L. G., Matsudo, S. M. M., \& Matsudo, V. K. R. (2004). Impacto da menarca nas variáveis antropométricas e neuromotoras da aptidão física, analisado longitudinalmente. Revista Brasileira de Ciência e Movimento, 12(2), 97-101.

Buligin, M. A. (1981). Models for improving a volleyballer's physical qualities. Soviet Sport Review, 16, 43-45.

Cabral, B. G. A., Cabral, S. A. T., Batista, G. R., Fernandes Filho, F., \& Knackfuss, M. I. (2008). Somatotipia e antropometria na seleção brasileira de voleibol. Motricidade, 4(1), 67-72.

Conselho da Europa (1990). Comissão para o desenvolvimento do desporto: Manual para os testes EUROFIT de aptidão física. Lisboa: Ministério da Educação.

Forwood, M. R., Baxter-Jones, A. D., Beck, T. J., Mirwald, R. L., Howard, A., \& Bailey, D. A. 
(2006). Physical activity and strength of the femoral neck during the adolescent growth spurt: A longitudinal analysis. Bone, 38(4), 576583. doi: 10.1016/j.bone.2005.09.021

Gabbett, T., Georgieff, B., \& Domrow, N. (2007). The use of physiological, anthropometric, and skill data to predict selection in a junior volleyball squad. Journal of Sports Science, 25(12), 1337-1344. doi: 10.1080/02640410601188777

Gallahue, D. L., \& Ozmun, J. C. (2005). Compreendendo o desenvolvimento motor: Bebês, Crianças, Adolescentes e Adultos. São Paulo: Phorte editora.

Gaya, A., \& Silva, G. (2007). Projeto Esporte Brasil PROESP - Bateria de Medidas e Testes Somatomotores do Proesp. Consultado em 12 de Março de 2010, a partir de http://www.proesp. ufrgs.br

Grave, K. C., \& Brown, T. (1976). Skeletal ossification and the adolescent growth spurt. American Journal of Orthodontics, 69(6), 611-619.

Kishali, N. F., Imamoglu, O., Katkat, D., Atan, T., \& Akyol, P. (2006). Effects of menstrual cycle on sports performance. International Journal of Neuroscience, 116(12), 1549-1563.

Latt, E., Jurimae, J., Haljaste, K., Cicchella, A., Purge, P., \& Jurimae, T. (2009). Longitudinal development of physical and performance parameters during biological maturation of young male swimmers. Perceptual \& Motor Skills, 108(1), 297-307. doi: 10.2466/pms.108.1.297307

Linhares, R. V., Matta, D., Lima, J. R. P., Dantas, P. S., Costa, M. B., \& Fernandes, J. (2009). Effects of sexual maturation on body composition, dermatoglyphics, somatotype and basic physical qualities of adolescents. Arquivos Brasileiros de Endocrinologia \& Metabologia, 53(1), 47-54. doi: 10.1590/S0004-27302009000100008

Little, B. B., \& Malina, R. M. (2007). Gene-environment interaction in skeletal maturity and body dimensions of urban Oaxaca Mestizo schoolchildren. Annals of Human Biology, 34(2), 216-225. doi: 10.1080/03014460601144011

Makarenko, L. P. (2001). Natação: seleção de talentos e iniciação desportiva. Porto Alegre: Artmed.

Malina, R. M. (2003). Crescimento, maturação e desempenho. In W. E. Garret Jr., \& D. T. Kirkendall (Eds.), A ciência do exercício e dos esportes (pp. 454-475). São Paulo: Artmed.

Malina, R. M., Bouchard, C., \& Bar-Or, O. (2004). Growth, maturation, and physical activity. Champaign: Human Kinetics.
Malina, R. M., Claessens, A. L., Van Aken, K., Thomis, M., Lefevre, J., Philippaerts, R., \& Beunen, G. P. (2006). Maturity offset in gymnasts: Application of a prediction equation. Medicine \& Science in Sports \& Exercise, 38(7), 1342-1347. doi: 10.1249/01.mss.0000227321. 61964.09

Malina, R., \& Bouchard, C. (2002). Atividade física do atleta jovem: Do crescimento à maturação. São Paulo: Rocca.

Marfell-Jones, M., Olds, T., Stewart, A., \& Carter, L. (2006). International standards for anthropometric assessment. Potchefstroom: International Society for the Advancement of Kinanthropometry.

Marins, J. C. B., \& Giannichi, R. S. (2003). Avaliação e prescrição de atividade física: Guia prático ( $3^{\mathrm{a}}$ ed.). Rio de Janeiro: Shape.

Matsudo, V. K. R., Araujo, T. L., Oliveira, L. C., \& Rodrigues, V. K. (2007). Há ciência na detecção de talentos? Diagnóstico \& Tratamento, 12, 196-199.

Mohamed, H., Vaeyens, R., Matthys, S., Multael, M., Lefevre, J., Lenoir, M., \& Philppaerts, R. (2009). Anthropometric and performance measures for the development of a talent detection and identification model in youth handball. Journal of Sports Science, 27(3), 257-266. doi: 10.1080/02640410802482417

Nedeljkovic, A., Mirkov, D. M., Kukolj, M., Ugarkovic, D., \& Jaric, S. (2007). Effect of maturation on the relationship between physical performance and body size. The Journal of Strength \& Conditioning Research, 21 (1), 245-250. doi: 10.1519/00124278-200702000-00044

Pearson, D. T., Naughton, G. A., \& Torode, M. (2006). Predictability of physiological testing and the role of maturation in talent identification for adolescent team sports. Journal of Science and Medicine in Sport, 9(4), 277-287. doi: 10.1016/j.jsams.2006.05.020

Perini, T. A., Oliveira, L. O., Ornellas, J. S., \& Oliveira, F. P. (2005). Cálculo do erro técnico de medição em antropometria. Revista Brasileira de Medicina do Esporte, 11(1), 81-85. doi: 10.1590/S1517-86922005000100009

Pyle, S. I., Waterhouse, A. M., \& Greulich, W. W. (1971). Attributes of the radiographic standard of reference for the National Health Examination Survey. American Journal of Physical Anthropology, 35(3), 331-337.

Roman, E. P., Ribeiro, R. R., Guerra Júnior, G., \& Barros Filho, A. A. (2009). Antropometry, sex- 
ual maturation and menarcheal age according to socioeconomic status of schoolgirls from Cascavel (PR). Revista da Associação Médica Brasileira, 55(3), 317-321. doi: 10.1590/S010442302009000300026
Schneider, P., \& Meyer, F. (2005). Avaliação antropométrica e da força muscular em nadadores pré-púberes e púberes. Revista Brasileira de Medicina do Esporte, 11 (4), 209-213. doi: 10.1590/S1517-86922005000400001

(c))EY-No Todo o conteúdo da revista Motricidade está licenciado sob a Creative Commons, exceto quando especificado em contrário e nos conteúdos retirados de outras fontes bibliográficas. 